\title{
Jugando a ser "YouTubers»: prácticas digitales para la prevención de la violencia de género
}

\author{
«Youtuber» izatera jolasten: \\ genero indarkeria prebenitzeko praktika digitalak \\ Playing «YouTubers»: \\ digital practices to prevent gender-based violence
}

Ester Villacampa Morales`, Sue Aran-Ramspott, Maddalena Fedele

Universitat Ramon Llull

RESUMEN: Recientes investigaciones señalan YouTube como una plataforma plenamente integrada en la vida mediática de los y las jóvenes y adolescentes. El presente proyecto pretende establecer si YouTube puede ser una herramienta para la prevención de la violencia de género entre este colectivo. Se aplica una aproximación cualitativa y participativa en dos grupos de edad y el diseño metodológico cuenta con una fase exploratoria en la que se realizan siete focus groups, una fase aplicada de Aprendizaje Servicio (APS) en que los participantes elaboran sus propias piezas para YouTube, y una fase de análisis de los vídeos resultantes.

PALABRAS CLAVE: Juventud; Alfabetización; Violencia machista; Aprendizaje-Servicio.

ABSTRACT: Recent studies point to YouTube as a fully integrated platform in the media life of youngsters and adolescents. This research project aims to establish if YouTube can be a tool for the prevention of gender violence among this collective. A qualitative and participatory approach is applied in two age groups of adolescents and the methodological design has an exploratory phase in which we carry out seven focus groups; an applied phase of Service Learning (APS), in which participants prepare their own pieces for YouTube; and a phase of analysis of the resulting videos.

KEYWORDS: Youth; Literacy; Gender violence; Service-Learning.

\footnotetext{
* Correspondencia a / Corresponding author: Ester Villacampa Morales. Facultad de Comunicación y Relaciones Internacionales Blanquerna. Plaça Joan Coromines 1 (0800 Barcelona) - estervm1@blanquerna.url.edu - https://orcid.org/0000-0002-7334-0298

Cómo citar / How to cite: Villacampa Morales, Ester; Aran-Ramspott, Sue; Fedele, Maddalena (2020). «Jugando a ser "YouTubers": prácticas digitales para la prevención de la violencia de género", Zer, 25(48), 287-308. (https://doi.org/10.1387/Zer.21570).

Recibido: 18 marzo, 2020; aceptado: 14 abril, 2020.

ISSN 1137-1102 - eISSN 1989-631X / (c) 2020 UPV/EHU

(c) (i) Esta obra está bajo una licencia

Creative Commons Atribución 4.0 Internacional
} 


\section{Introducción}

El análisis de los riesgos y de las posibilidades que supone YouTube para los adolescentes se está consolidando como un objeto de estudio específico dentro de la investigación sobre el impacto de las redes sociales en las generaciones digitales. Desde las primeras investigaciones que señalaban la creciente penetración de dichas redes entre la juventud (Buckingham, 2008; Livingstone, 2008; Boyd, 2014), actualmente se confirma cómo el consumo audiovisual conserva su soberanía (Gewerc, Fraga y Rodes, 2017) pero en la modalidad a demanda que permiten las nuevas plataformas, lideradas por YouTube (Fernández-de-Arroyabe-Olaortua, LazkanoArrillaga y Eguskiza-Sesumaga, 2018: 62). En España, existen diversos estudios que confirman que los y las adolescentes efectúan un uso de YouTube eminentemente como entretenimiento (Viñals, Abad y Aguilar, 2014); donde se observan diferencias es en el lugar que ocupan a continuación otras funciones. Funciones como las de (auto)aprendizaje o información, socialización y la de comunidad virtual, en tanto que sensación de pertenencia a una cultura digital juvenil (Aran-Ramspott, Fedele y Tarragó, 2018; Pires, Masanet y Scolari, 2019).

En relación a la variable de género, se coincide en que tanto chicos como chicas son más consumidores que prosumidores, pero esta característica común sobre un tipo de consumo más tradicional tiene su contrapartida: al analizar con detalle los contenidos que se siguen en YouTube, existe un sesgo de género en la preferencia de los hombres por los juegos, así como por la belleza y moda por parte de las mujeres (Linares-Bahillo, Aristegui-Fradua y Beloki-Marañón, 2018). Este sesgo también se manifiesta en la desigual repercusión de los y las YouTubers en cuanto a número de seguidores y visualizaciones (Wotanis y McMillan, 2014). Sin embargo, la plataforma también se ha considerado habitualmente como un espacio para la exploración y representación de identidades de género no normativas, al menos por lo que respecta a la presencia y visibilidad del colectivo LGTBQ (Pérez-Torres, Pastor-Ruiz y Abarrou-Ben-Boubaker, 2018; Tortajada, Caballero-Gálvez y Willem, 2019). Si bien no es la plataforma preferida para un uso eminentemente activista, sí que se detectan en ella presencia de representaciones que rompen la lógica postfeminista (Caballero-Gálvez, Tortajada y Willem, 2017) e incluso discursos feministas (Araüna, Tortajada y Willem, 2019).

Los estereotipos de género, que persisten mayoritariamente entre las nuevas generaciones (Pibernat, 2017), forman parte de la estructura menos evidente del denominado iceberg de la violencia. En España, sólo se ha estudiado la punta del iceberg, ese $10 \%$ de las mujeres víctimas de maltrato que se llega a conocer (Fernández Alonso, 2003: 37). Los estereotipos suelen retroalimentar otras formas de violencia simbólica o estructural menos evidentes pero que están presentes en la recurrencia de los chistes sexistas, en el lenguaje discriminatorio, en los micromachismos y, también, en los mecanismos de invisibilización. Los estereotipos discri- 
minatorios, como recoge el manual periodístico de Amnistía internacional (2009), «se refuerzan en la juventud y la madurez a través de formas de control perversas, impunidad ante el acoso sexual contra las mujeres, dominio de los espacios físicos, falta de protección oficial, discriminación laboral y salarial, sobrecarga de tareas en la economía reproductiva, etc.». Como señala este mismo organismo (AI, 2019), la violencia contra las mujeres es la consecuencia más extendida de la discriminación de género. Y no hay que olvidar que así como las redes sociales favorecen una mayor visibilidad de la violencia social, también emergen nuevas formas de violencia, como el ciberacoso.

De todo ello se desprende la importancia de una educación que desde las más tempranas edades colabore en la prevención de cualquier forma de discriminación y de violencia, en el caso que nos ocupa de la violencia de género, así como del recurso a los contenidos audiovisuales, de las fuentes de información y de las redes sociales como herramientas de análisis, debate, concienciación y de creación de referentes e imaginarios más igualitarios. Es la alfabetización mediática (Buckingham, 2003) la disciplina académica que promueve el desarrollo e integración de las competencias mediáticas en el currículum educativo con el fin de fomentar una lectura crítica de los medios de comunicación entre los más jóvenes. En el ecosistema mediático actual, se hace necesario actualizar el concepto para incluir también aquellos aprendizajes informales que los adolescentes adquieren en el marco de la cultura participativa, por lo que algunos autores sugieren el término alfabetización transmediática (Scolari, 2016).

Precisamente YouTube y otras redes sociales pueden ser herramientas para estos tipos de aprendizajes informales entre los más jóvenes, gracias a los mecanismos de participación y prosumición que las caracterizan, que podrían hacer más eficaz el aprendizaje en torno a la violencia de género. En este sentido, cabe mencionar los estudios sobre la eficacia de las campañas de prevención de riesgos (drogas, alcohol, seguridad vial, enfermedades de transmisión sexual, prevención de embarazos no deseados...) dirigidas a adolescentes y jóvenes, ya que «las investigaciones pedagógicas en prevención apuntan a una eficacia mayor de los procesos preventivos en los que los y las destinatarios son protagonistas de su proceso de aprendizaje» (Obra Social «la Caixa», 2006: 6). Investigaciones como las de López-Vidales y Gómez-Rubio (2015) señalan la dificultad de que las campañas institucionales logren una aceptación amplia entre el público adolescente. Nuestra experiencia previa en este tipo de campañas (Aran y de Puig, 2011) indica que narrativas y recursos retóricos de carácter impositivo generan un mayor rechazo entre los jóvenes a quienes se dirigen. De manera similar, podemos resumir que a mayor preocupación y expresión de los legítimos miedos adultos, mayor distanciamiento se activa por parte de los jóvenes receptores. Esta dificultad aparece como un motivo importante para otorgar a los propios adolescentes y jóvenes un rol protagonista en el diseño y ejecución de contenidos, que, en el proyecto que nos ocupa, se dirige a la prevención de la violencia de gé- 
nero. Por tanto, no se trata únicamente de «empoderar» a los sujetos, sino de hacerlos creadores de los propios mensajes.

Y en términos de eficacia comunicativa, todavía hay otro dato que colabora en la decisión de integrar a los adolescentes desde la misma génesis de un movimiento de responsabilización individual que no se base en la normatividad: los adolescentes consumen el doble los vídeos de adolescentes que de otros autores, y los de los YouTubers son los de mayor impacto (Montes-Vozmediano, García-Jiménez y Menor-Sendra, 2018: 68). La función identitaria, los mecanismos de identificación e incluso admiración que ejercen determinados jóvenes YouTubers, así como la función de socialización que el medio permite, son un tipo de usos mediáticos que pueden colaborar con fines no necesariamente comerciales. Existen experiencias previas de YouTubers que, conscientes de su rol como referentes entre sus jóvenes audiencias (Westenberg, 2016), se han implicado en la prevención de la violencia. Una iniciativa interesante es el programa educativo para jóvenes de 12 a 18 años \#SomosMás, impulsado por la propia plataforma YouTube (en colaboración con diversas instituciones) y que tiene como objetivo combatir el odio y el radicalismo. Si bien los y las YouTubers participan activamente, esta experiencia propone también la realización de vídeos por parte de los participantes.

Por todo lo expuesto, se ha realizado el presente proyecto de investigación aplicada y participativa entre estudiantes barceloneses de entre 15 y 20 años (adolescencia mediana y tardía), procedentes de institutos y de la universidad, poniendo el foco en las posibilidades de un aprendizaje basado en el consumo y la creación en YouTube (prosumición) que favorezca unas relaciones de género inclusivas y la prevención de la violencia de género.

El proyecto plantea la siguiente pregunta de investigación: ¿Puede ser YouTube para las nuevas generaciones un instrumento a favor de la sensibilización y prevención de la violencia de género? Como preguntas secundarias se despliegan las siguientes: ¿Qué conocimientos tienen los y las jóvenes y adolescentes sobre la violencia de género?, ¿Reconocen la presencia de violencia de género en los discursos (contenidos y/o formas expresivas) que promueven los YouTubers?, ¿Qué temáticas, YouTubers y fórmulas expresivas identifican con la prevención de la violencia, concretamente de género?, ¿Qué rol atribuyen las nuevas generaciones a los y las YouTubers, en particular qué rol consideran que ejercen en la promoción y/o prevención de la violencia de género?, ¿Qué tipos de contenidos para YouTube sobre la prevención de la violencia de género pueden crear los propios adolescentes?

El objetivo principal de este proyecto es, por tanto, promover la prosumición entre los y las adolescentes y comprobar cómo YouTube puede actuar como herra- 
mienta de prevención de la violencia de género entre este colectivo. Este objetivo general se articula en los siguientes sub-objetivos:

1. Entender cómo los y las adolescentes perciben y reconocen en YouTube la presencia de la violencia de género.

2. Proporcionar a adolescentes y jóvenes la base teórica para conceptualizar la violencia de género a partir de ejemplos presentes en YouTube.

3. Establecer cómo una acción educomunicativa puede aprovechar el consumo y la creación en YouTube para promover modelos mentales o formas de actuación que colaboren en la prevención de la violencia de género entre el colectivo adolescente.

4. Facilitar instrumentos conceptuales y creativos para el abordaje de la prevención de la violencia de género, como proyectos audiovisuales en formato YouTube.

5. Analizar los modelos y los formatos que los propios adolescentes proponen para la prevención de la violencia de género.

\section{Metodología}

Este proyecto es una investigación aplicada que, a partir de una aproximación cualitativa, contempla tres grandes fases y diversas técnicas de investigación (figura 1): 1) una fase exploratoria, consistente en el análisis de las percepciones juveniles respecto a la violencia de género y su presencia en YouTube, realizado mediante siete focus groups (Sub-objetivo 1); 2) una fase aplicada de Aprendizaje Servicio (APS) (Arandia, Alonso y Martínez, 2010; Annette, 2005), consistente en el diseño e implementación de seis talleres educomunicativos, en que los participantes guionizan y elaboran sus propias piezas para YouTube (Sub-objetivos 2, 3 y 4) y que se dan a conocer en la comunidad; 3) una fase de análisis de contenido de los vídeos realizados por los y las participantes (Sub-objetivo 5).

El trabajo de campo se realizó entre los meses de octubre y diciembre de 2019, en un centro universitario y tres centros de educación secundaria de Barcelona. Tanto los grupos de discusión como los talleres se llevaron a cabo en los respectivos centros educativos con la presencia de dos investigadoras y del profesor o tutor de cada grupo, previa autorización por escrito de todos los participantes o de sus tutores legales en el caso de menores.

El estudio contó con la participación de 42 participantes en la primera fase y 106 en la segunda, todos ellos adolescentes y jóvenes de entre 15 y 20 años. En particular, se trabajó con dos franjas de edad, la de 15-16 años (correspondiente a la adolescencia mediana/central) y la de 19-20 años (correspondiente a la adolescencia tardía). 


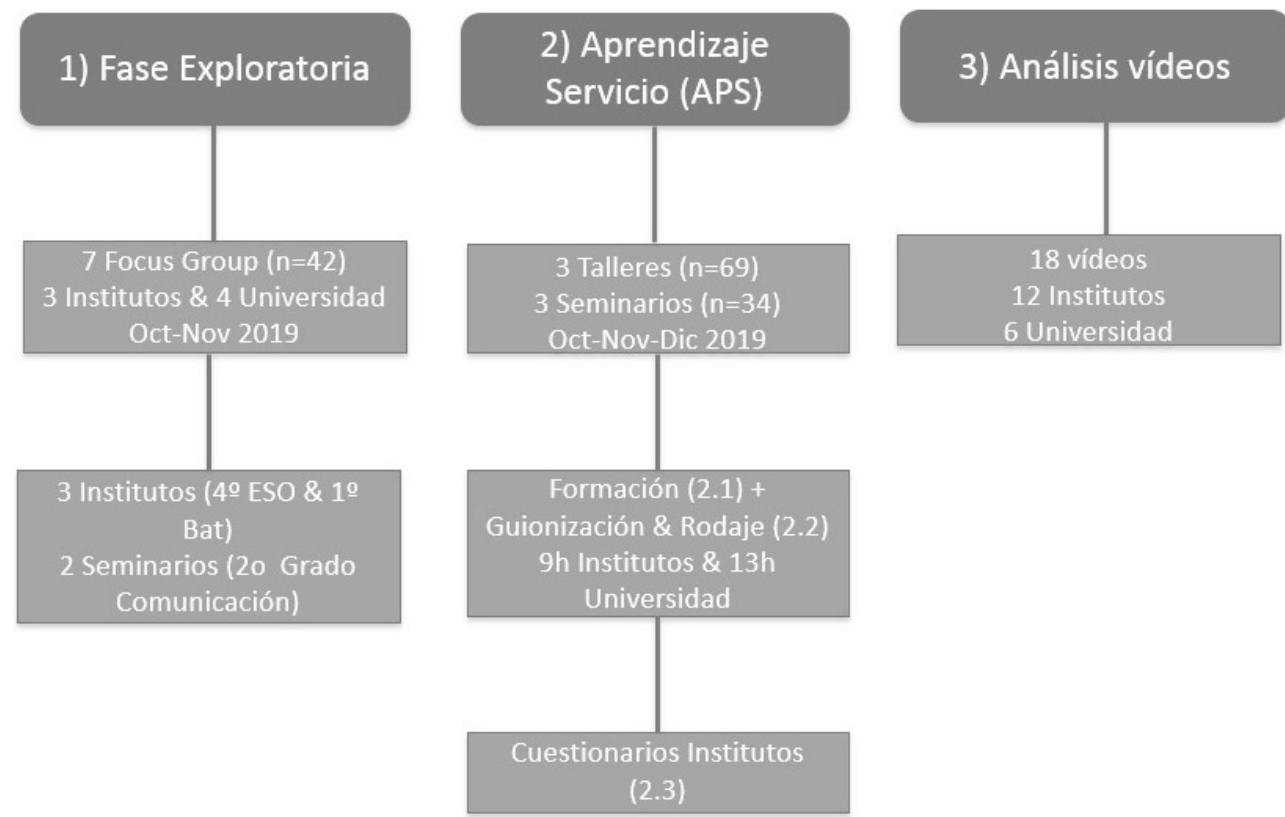

Fuente: elaboración propia.

Figura 1

Diseño metodológico

\subsection{FASE EXPLORATORIA: LOS FOCUS GROUPS}

Los focus groups, organizados a partir de preguntas semiestructuradas, sirvieron para ahondar en los usos y preferencias de los y las adolescentes en relación a YouTube y a los YouTubers, así como en la propia noción de violencia de género, y su percepción de esta violencia en YouTube y en YouTubers concretos.

Se realizaron siete focus groups de seis participantes cada uno (respetando la paridad de género), con un total de 42 participantes, de acuerdo a la siguiente distribución: tres en institutos (dos de ellos entre estudiantes de $4 .^{\circ}$ de la ESO, y uno entre estudiantes de $1 .^{\circ}$ de Bachillerato), y cuatro entre estudiantes de $2 .^{\circ}$ del Grado en Comunicación Audiovisual. De los tres institutos seleccionados, dos son de titularidad pública y están ubicados en barrios de renta media-baja y muy baja respectivamente, mientras que el tercero es concertado y se sitúa en un barrio de renta baja según datos de 2017 (Ajuntament de Barcelona, 2017). La facultad es de titularidad privada, pero depende de una fundación sin ánimo de lucro y está ubicada en un barrio de renta baja. 
El registro de los focus groups se realizó en los propios centros educativos, mediante grabación de audio, y el seguimiento y codificación posterior se llevó a cabo por parte de dos investigadoras, con el soporte del programa Atlas.ti. Los resultados de las contribuciones de los grupos de discusión se identifican en este artículo de la siguiente manera: número del focus group (FG1 hasta FG7) + etapa educativa (instituto/universidad) + participante (chico/chica) + número de participante (por orden de intervención).

\subsection{Fase aplicada: los talleres educomunicativos}

La segunda fase contó con las siguientes sub-fases, de manera que el ciclo concluyera como una experiencia guiada por especialistas en educación mediática con una perspectiva de género, en la que las piezas audiovisuales resultantes fueron dirigidas y protagonizadas por los y las jóvenes participantes:

— Sub-fase 2.1) Formación educomunicativa sobre análisis filmico de vídeos de YouTube en las aulas, incluyendo tanto contenidos con una perspectiva estereotipada de la masculinidad y de la feminidad como desde posiciones más igualitarias e inclusivas.

- Sub-fase 2.2) Guionización, rodaje e indicaciones de montaje de piezas audiovisuales para YouTube por parte de los participantes (prosumición).

- Sub-fase 2.3) Cuestionario sobre los resultados del proceso.

En esta fase, participaron 103 adolescentes y jóvenes, siendo 69 estudiantes de secundaria y 34 universitarios. Las 72 chicas y 31 chicos, organizados en 15 grupos (nueve en secundaria y seis en la universidad), realizaron, en total, 15 piezas audiovisuales para YouTube.

El diseño de contenidos de los talleres ha sido fruto de la colaboración entre las investigadoras del proyecto y las integrantes de Drac Màgic, entidad no lucrativa con más de 45 años de experiencia en la realización de talleres de educación mediática.

Se realizaron seis sesiones tanto en los tres institutos participantes (talleres) como en los tres grupos reducidos de alumnos (seminarios) de la facultad. La distinción terminológica entre talleres y seminarios corresponde respectivamente a la implementación en los institutos (a cargo de la entidad colaboradora) y en la universidad (a cargo del profesorado de la Facultad de comunicación y Relaciones Internacionales Blanquerna-URL).

La duración de la acción participativa (Sub-fases 2.1 y 2.2) ha sido de nueve horas en los institutos y de trece horas en la universidad, de acuerdo a las dinámicas internas de la duración de las clases. La (auto)evaluación mediante cuestionarios (Sub-fase 2.3) $(n=70)$ ha comportado media hora más final en cada grupo. 
Sub-fase 2.1. Formación en análisis fílmico

Se dedicó la primera sesión de los talleres a la elaboración de un mapa del consumo mediático de los jóvenes y adolescentes, basado en la puesta en común de los atributos más destacados de los YouTubers con mayor seguimiento, de los principales temas abordados y de las características audiovisuales de sus vídeos. La segunda consistió en el visionado de diversos ejemplos de influencers en relación a la perspectiva de género y la posterior evaluación sobre las estrategias y características performativas de los mismos, así como los recursos retóricos y de formato empleados (humor, sátira, entretenimiento, crítica, conformidad...), con el fin de reflexionar sobre las posibilidades de YouTube como instrumento de reafirmación o de crítica social.

\section{Sub-fase 2.2. Aprendizaje-Servicio (APS)}

En esta fase se introdujeron y abordaron los siguientes contenidos y procesos: ideación y guionización de los vídeos, rodaje, edición y puesta en común con un visionado final. En el marco de la metodología pedagógica del Aprendizaje Servicio (APS) (Eyler y Giles, 1999; Annette, 2005; Puig, 2012), se orientaron las competencias mediáticas (Ferrés y Piscitelli, 2012) hacia la temática principal de la investigación, de manera que el aprendizaje experimental se integrara en el desarrollo de una responsabilidad cívica y educación en valores de los estudiantes hacia el propio colectivo de adolescentes y jóvenes (Puig, 2012). Se ha seguido la definición de Aprendizaje Servicio de Puig, Martín y Batlle (2010: 20) según la cual la propuesta educativa combina procesos de aprendizaje y de servicio a la comunidad «en un único proyecto bien articulado en el que los participantes aprenden a la vez que trabajan en necesidades reales del entorno con la finalidad de mejorarlo». Como se detalla en las conclusiones, el modelo de aprendizaje y los resultados de la presente propuesta son accesibles de manera gratuita a la comunidad.

La temática debía girar alrededor de las formas de violencia de género y de su prevención. Se dieron las siguientes indicaciones, que suelen ser condicionantes de producción básicos a seguir: duración aproximada (entre 1 y 5 minutos), recursos de rodaje (cámara digital, trípode, microfonía y soporte para registro del sonido directo aportados por el equipo de investigación o por la entidad colaboradora), recursos de puesta en escena (a criterio de los estudiantes), casting e interpretación (a cargo de los estudiantes) y localizaciones (dentro o en las inmediaciones de cada centro).

Los estudiantes se organizaron en grupos de entre 5 y 9 integrantes, intentando respetar la paridad de género. Aún así, tanto en institutos como en la facultad la proporción de mujeres es mayor (llegando a ser mucho mayor en el caso de algunos institutos). Esto podría explicarse por el hecho de que los talleres se han desarrollado 
en el marco de asignaturas optativas de disciplinas artísticas o dentro de itinerarios sociales y/o artísticos, ambos mayormente cursados por estudiantes de sexo femenino. Los grados de comunicación, a su vez, también están mayoritariamente ocupados por mujeres.

Respecto a la composición de cada grupo, cabe mencionar que el número de integrantes por grupo tuvo que ver con el número total de personas en el aula de cada centro, menor entre los universitarios.

Como se ha mencionado previamente, se realizaron 15 piezas audiovisuales (una por grupo), nueve en los institutos (duración media de 1'30”) y seis en la universidad (duración media de 4'30”).

\section{Sub-fase 2.3. Cuestionario}

La última fase del proceso de la acción participativa responde a la realización de un cuestionario de valoración por parte de los estudiantes de instituto con el fin de establecer los aprendizajes adquiridos durante el proceso de trabajo. El cuestionario consta de seis preguntas, es anónimo (con la excepción del centro y género del estudiante) y se administró en formato papel una semana después de la finalización de la experiencia. Su realización no se ha considerado necesaria en el caso de los seminarios puesto que, al tratarse de estudiantes de comunicación, el programa ha sido alterado y la formación audiovisual ha sido previamente adquirida por otras vías.

\subsection{FASE DE ANÁlisis de CONTENIDO}

El modelo de análisis de contenido efectuado sobre los vídeos realizados por los estudiantes se ha basado parcialmente en el modelo propuesto por Montes-Vozmediano et al. (2018) al cual se han incorporado referentes como Simonsen (2012).

En concreto, se han analizado dos grupos de variables:

a) Variables descriptivas: tipo de centro, título del vídeo, duración, autoría (número y género de los integrantes).

b) Variables temático-estructurales, en relación a la presencia de la violencia de género:

- género o formato del vídeo (vlogs, interpretaciones musicales, tutoriales, momentos de YouTube, artísticos y líricos, declaraciones políticas, ficción y sketches, parodias, y entrevistas y reportajes, según Simonsen, 2012); 
- orientación del discurso - positiva (se trata de resolver un problema), negativa (se rechazan soluciones al problema sin aportar alternativas), neutra o indeterminada (únicamente se explica el hecho)-;

- estructura e interpelación al usuario/a (declarativa - vídeos con modalidad aséptica-, imperativa — vídeos que incluyen órdenes que mueven a la acción- o interrogativa/reflexiva — vídeos que plantean preguntas o que mueven la conciencia-, basada en Halliday, 2004);

- contenidos relacionados con la prevención de la violencia de género (tipo de violencia, tipo de personajes y narrador/a, espacio — público o privado-y temporalidad de la representación).

\section{Análisis y resultados}

A continuación detallamos los resultados principales obtenidos en el desarrollo del proyecto, atendiendo principalmente a la fase exploratoria (análisis de los focus groups) y a la fase de análisis de contenido (análisis de los vídeos realizados por los estudiantes), con el fin de establecer un diálogo entre ambos procesos, en lo que refiere a la variable de género, así como en la conceptualización y abordaje de la violencia y en especial la de carácter machista y su prevención.

\subsection{Resultados de los FOCUS GROUPS}

\subsubsection{Usos, contenidos y YouTubers mayoritarios}

En consonancia con investigaciones previas, los resultados muestran un uso preferente de YouTube con funciones de entretenimiento y aprendizaje informal, por ejemplo, en el consumo de lo que los participantes denominan «vídeos de curiosidades». Algunos participantes utilizan YouTube para «estar al día», uso que se corresponde con la variable de comunidad virtual. El uso de la plataforma como vínculo virtual entre iguales y/o de socialización es menor, puesto que ambos grupos de edad señalan Instagram como su red social favorita para este fin.

En cuanto al tipo de contenido consumido, destacan la música y el entretenimiento en ambos sexos, seguidos de entrevistas y reportajes. Algunos participantes afirman haber sustituido plenamente la televisión tradicional por YouTube y por otras plataformas como Netflix. Si bien este tipo de consumo no presenta distinciones de género, sí existe, sobre todo entre los más jóvenes, una preferencia de los chicos por el gaming y de las chicas por vlogs (de moda, de viajes y de fotografia).

De acuerdo con ello, un nombre hacia el que la mayoría de participantes adolescentes muestran agrado es el de AuronPlay, puesto que el YouTuber rea- 
liza contenido de entretenimiento y comedia. En segundo lugar, aparecen mencionados elRubius, Wismichu, TheGrefg y PewdePie, a los que atribuyen indistintamente características positivas y negativas. De hecho, en la franja de edad entre los 15 y 16 años, los participantes empiezan a desvincularse de estos YouTubers con afirmaciones del tipo: "Yo en $6 .^{\circ}$ de primaria sí seguía a elRubius pero ahora me parece infantil y repetitivo» (FG1, Instituto, Chico1). Por su parte, los estudiantes universitarios se desvinculan completamente de todos ellos (incluido AuronPlay) y desdeñan la conversación alegando que el público potencial de estos YouTubers son niños. Además, insisten en la responsabilidad moral de los YouTubers en tanto que ejemplo para sus audiencias de menor edad. Si bien los universitarios siguen a algunos YouTubers, realizan un tipo de consumo más especializado, donde no prima la persona sino el interés personal por una temática. De acuerdo con las características sociodemográficas de Barcelona, en el barrio con más alto porcentaje de población migrada, los participantes de este instituto manifiestan gustos y aversiones tanto hacia YouTubers españoles como hacia los de sus propios países de origen (mayoritariamente latinoamericanos y en menor medida del norte de África).

En cuanto a los motivos de desagrado, aparecen la falsedad, la hipocresía, el clickbait, la exageración y la polémica: «Es que los títulos son como súper exagerados y luego no va de eso el vídeo» (FG3, Instituto, Chico1). La falsedad se vincula con YouTubers mujeres como Dulceida, Paula Gonu y, en menor medida, Marina Yers, mientras que la polémica acompaña a Dallas Review. Hay un reconocimiento por parte de los participantes de que son las políticas de monetización de YouTube las que contribuyen a la construcción de una imagen cuanto menos idealizada, así como a este reclamo excesivo de atención por parte de los YouTubers: "Si les sigue funcionando lo seguirán haciendo, porque ellos ganan dinero de estas polémicas» (FG5, Universidad, Chica 2).

\subsubsection{Violencia en YouTube}

Cuando los participantes son interpelados sobre la violencia en YouTube, afloran los casos mediáticos de Reset (que dio dinero a un vagabundo a cambio de comerse una galleta con pasta de dientes) y de MrGranBomba (que recibió un guantazo tras insultar a un transeúnte al grito de "cara anchoa»). Son también los nombres de Logan Paul y Dallas Review los que aparecen frecuentemente vinculados a conductas y casos de violencia. Entre estos casos, los participantes manifiestan haber encontrado maltrato animal, maltrato a niños y/o ancianos, conductas de riesgo hacia la propia integridad y contenidos racistas y sexistas. De acuerdo al importante origen diverso de los participantes en el barrio con más alto porcentaje de población migrada, se detecta una mayor preocupación por las formas de violencia racistas. 
Sin embargo, la mayoría de los participantes afirma que la violencia es mucho más explícita en Instagram debido a las políticas de restricción de contenido en YouTube, al cual atribuyen un público de edad cada vez menor. Esta política de empresa hace que emerjan las críticas a la censura en YouTube, particularmente en su vertiente sexual: «tal como está ahora YouTube lo censuran todo (FG4, Universidad, Chico2); "censuran pezones y lo que tienen que censurar, nada» (FG2, Instituto, Chica3).

De igual manera que la violencia contra animales aparece como la más mencionada, los participantes también reconocen la sensibilidad de algunos YouTubers en contra de ella y, en general, a favor del veganismo y de la conciencia ecológica. Como veremos más adelante, la sensibilidad hacia la violencia de género cuesta más de identificar con nombres propios. Por lo que respecta a la reacción hacia todo tipo de violencia, se da casi mayoritariamente un uso pasivo o reactivo de la plataforma puesto que la solución, según interpretan los participantes, consiste en "quitar el vídeo». Sin embargo, en casos extremos, los estudiantes denuncian el contenido a YouTube, aunque manifiestan sus reservas en cuanto a la utilidad de dicha práctica, puesto que hacen falta muchas denuncias para que se emprendan acciones.

\subsubsection{Violencia de género}

En relación a la violencia de género, en los institutos los estudiantes la definen no tanto conceptualmente sino a través de situaciones características. Por ejemplo, se refieren al maltrato físico o psicológico, a la violencia sexual, el acoso callejero, los micromachismos (no siempre usando este término), la discriminación por razón de género y a la cosificación de la mujer: "Hablar de las mujeres como si fueran objetos» (FG3, Instituto, Chico1). Entre los estudiantes universitarios, el maltrato cobra más importancia y se incorpora explícitamente en la propia conceptualización que hacen de la violencia de género: «El maltrato o discriminación de un género hacia otro por el hecho de ser de ese género» (FG5, Universidad, Chica2). Es destacable la concepción entre algunos de los participantes universitarios de que la violencia de género funciona en ambas direcciones.

Resulta dificil para la mayoría de estudiantes identificar nombres de YouTubers que se refieran a la violencia de género tanto desde una presencia no problemática como desde el rechazo o la crítica, aunque en más de una ocasión afirman que «hay muchos». Así como en el caso de la incentivación de la violencia hay un acuerdo mayoritario en mencionar a Dalas Review (aunque se le censura más por «polémico» que por los sonados casos de violencia de género en los que se ha visto envuelto), el rechazo o la prevención se concentran más en vídeos específicos que en canales o YouTubers identificados con dicha posición crítica. Sin embargo, tres nombres han aparecido en más de una ocasión y en el siguiente orden: Luc Loren, Devermut y 
Herrejón. Los tres nombres han aparecido como YouTubers comprometidos contra la violencia de género y a favor de los derechos de la comunidad LGTBQ. Como han demostrado otras investigaciones (Linares-Bahillo, Aristegui-Fradua y BelokiMarañón, 2019), solo algunas chicas sugieren nombres en esta línea, aunque entre los estudiantes universitarios hay un mayor reconocimiento de los mismos también por parte de los chicos.

Existe, además, cierta percepción entre algunos participantes de que actualmente hablar de feminismo en YouTube aporta visitas y aunque se apoya que así se haga, los estudiantes distinguen entre los YouTubers que merecen confianza (los anteriormente mencionados) y los que les resultan menos auténticos o sinceros (por ejemplo, Dulceida). En cuanto a los métodos y formatos para abordar la temática en YouTube, los estudiantes abogan por el «monólogo» por su capacidad de ser claro y conciso, las entrevistas y la introducción de la perspectiva de género en vídeos de otras temáticas. En cuanto al humor, la mayoría manifiesta reparos y solo después de debatirlo con compañeros lo aceptan como una estrategia lícita para sensibilizar contra la violencia de género, siempre que «esté muy bien hecho» (FG7, Universidad, Chico3).

\subsection{ANÁlisis De CONTENIDO DE LOS VídeOS REALIZADOS POR EL ALUMNADO}

El análisis de contenido de las piezas realizadas por los estudiantes se comenta a continuación, diferenciando de acuerdo a la metodología aplicada las mencionadas variables descriptivas y temático-estructurales. 


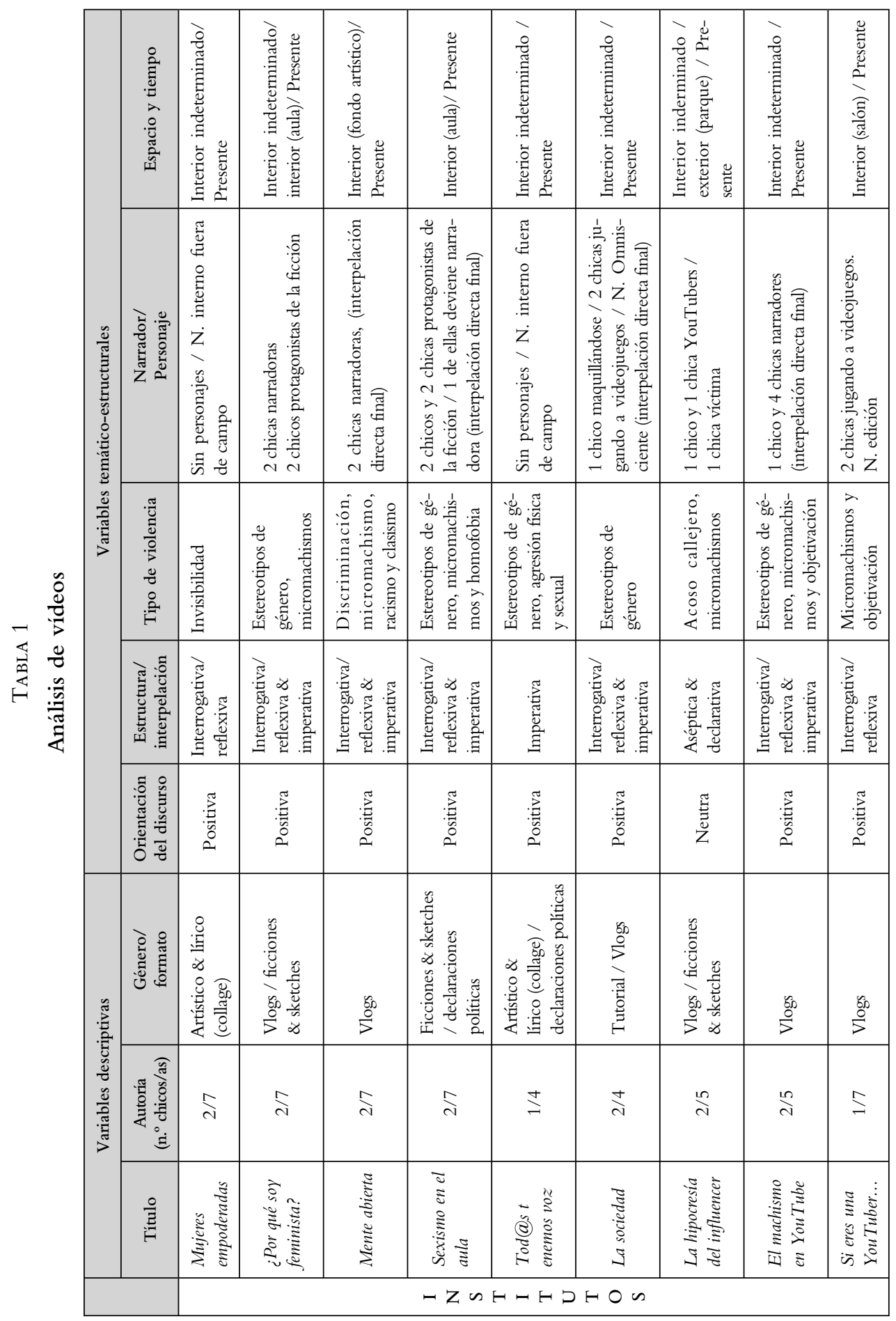


Jugando a ser "YouTubers": prácticas digitales para la prevención de la violencia de género

\begin{tabular}{|c|c|c|c|c|c|c|c|}
\hline \multirow{5}{*}{ 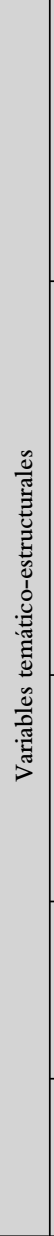 } & 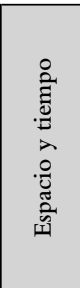 & 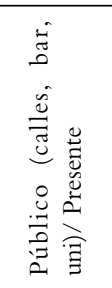 & 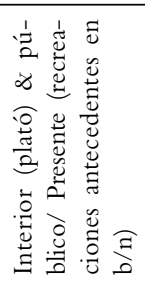 & 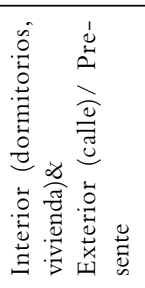 & 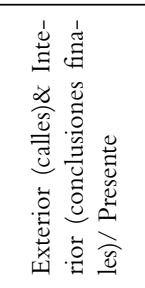 & 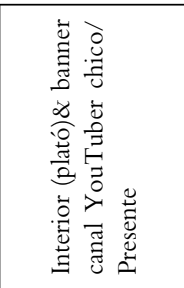 & 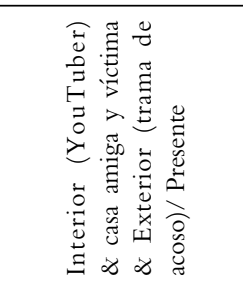 \\
\hline & 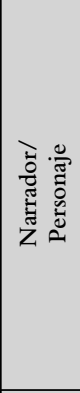 & 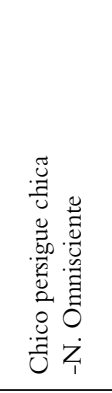 & 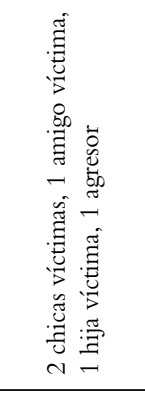 & 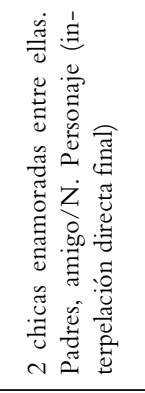 & 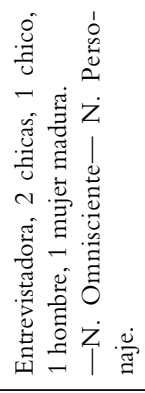 & 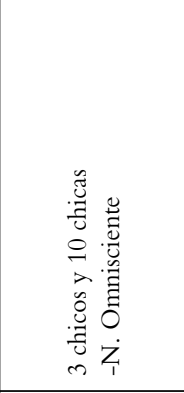 & 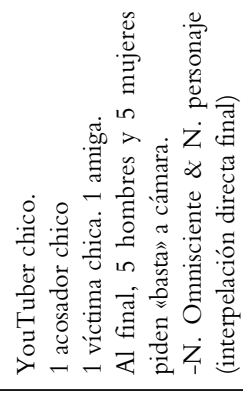 \\
\hline & 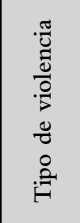 & 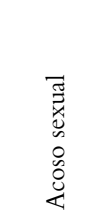 & 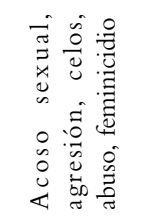 & 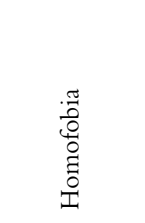 & 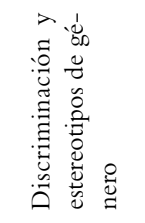 & 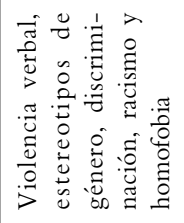 & 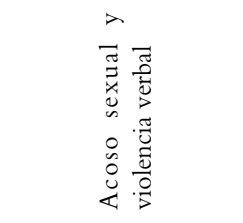 \\
\hline & 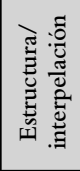 & 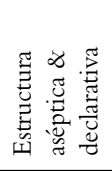 & 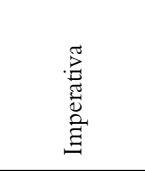 & 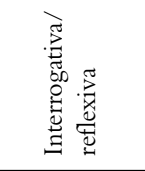 & 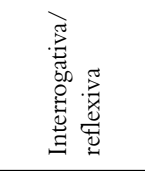 & 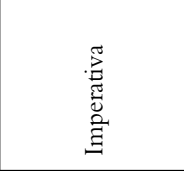 & 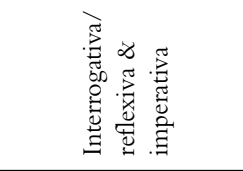 \\
\hline & 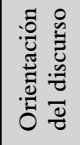 & 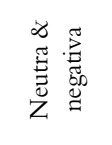 & 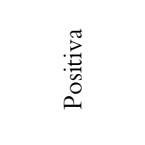 & $\begin{array}{l}\sum_{0}^{0} \\
0 \\
0\end{array}$ & 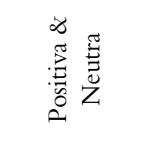 & 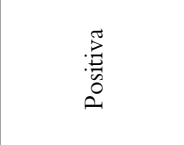 & 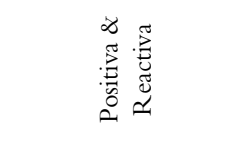 \\
\hline \multirow{3}{*}{ 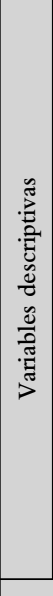 } & 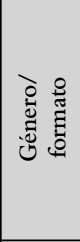 & 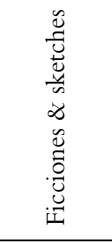 & 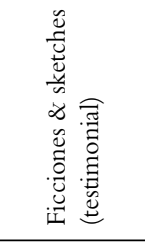 & $\stackrel{\substack{80 \\
>}}{\stackrel{8}{8}}$ & 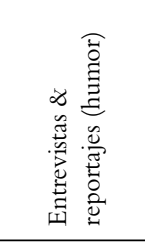 & 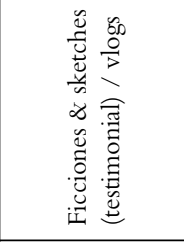 & 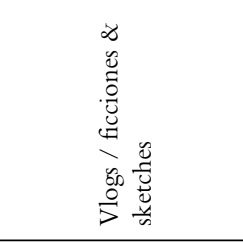 \\
\hline & 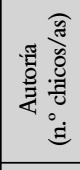 & $\stackrel{\curvearrowright}{\lambda}$ & $\frac{f}{f}$ & $\stackrel{m}{m}$ & $\stackrel{y}{a}$ & $\stackrel{\Delta}{\Delta}$ & $\stackrel{\sim}{\lambda}$ \\
\hline & $\underset{⿱ 乛}{\stackrel{D}{Z}}$ & 产 & 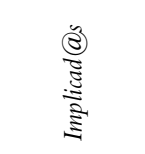 & 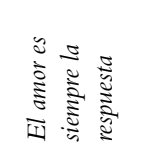 & 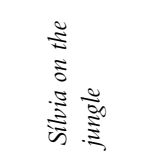 & 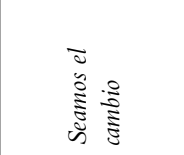 & 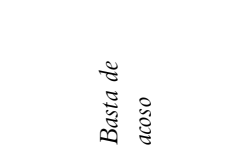 \\
\hline & & \multicolumn{6}{|c|}{$\varangle \theta$} \\
\hline
\end{tabular}




\subsubsection{Variables descriptivas}

Por lo que se refiere a los títulos, los vídeos mantienen cierto equilibrio entre aquellos enunciados de carácter descriptivo (por ejemplo, "Mujeres empoderadas», «Sexismo en el aula», "El machismo en YouTube», "Acoso»...) respecto a aquellos que implican ("Tod@s tenemos voz», «Implicad@s», "Seamos el cambio») o interpelan («Si eres una YouTuber...», "¿Por qué soy feminista?», "Basta de acoso») de manera más directa a un colectivo, al público o a la sociedad en general.

La intención de los títulos señala en la mayoría de casos una coherencia con variables temáticas y estructurales, tanto en relación a la presencia de la violencia de género como a la orientación del discurso, la estructura (o interpelación) y, con menor evidencia, el género o formato («Sílvia on the jungle» es el ejemplo más claro de la intención y estructura humorística de la pieza).

Respecto a la autoría, la gran mayoría de los vídeos han sido fruto de un trabajo grupal de entre cinco y nueve autores, siendo el número más reducido entre los universitarios, la mayoría integrados en grupos de seis personas.

\subsubsection{Variables temáticas y estructurales, en relación a la presencia de la violencia de género}

En primer lugar, los adolescentes y jóvenes presentan cierta diversidad en cuanto al formato del vídeo orientado a la plataforma YouTube. De acuerdo con las posiciones adoptadas en los grupos de discusión y con el encargo realizado, la mayoría de grupos han optado por el formato $v \log$, caracterizado principalmente por la presencia de un autor-protagonista que expone un tema u opinión en primera persona y que se dirige a su audiencia de forma directa. Si bien este formato permite lanzar ideas claras y concisas, algunos vídeos incorporan otros recursos con el fin de ilustrar su discurso, como por ejemplo el diálogo («Mente abierta») o los fragmentos ficcionados («¿Por qué soy feminista?», "La hipocresía del influencer», «Basta de acoso», $\mathrm{y}$ «Seamos el cambio»). Cabe destacar, sin embargo, el formato testimonial que adopta este último que, junto a «Implicad@s», se inspira en las campañas institucionales para la prevención de la violencia de género. Uno de los pocos casos en que la ficción se ha utilizado como formato único coincide con aquel de estructura declarativa y orientación del discurso negativa ("Acoso»). Mientras entre los universitarios se encuentra el único caso del género referido como «entrevistas y reportajes» («Sílvia on the jungle»), entre los adolescentes destacan dos ejemplos de formato artístico mediante la técnica del collage. Si bien en «Mujeres empoderadas» dicha técnica se acompaña de un discurso interrogativo, en «Tod@s tenemos voz»la estructura imperativa inclina la balanza hacia un formato más próximo a las «declaraciones políticas». De estos datos se puede derivar que no hay un afán lúdico de las propuestas audiovisuales del alumnado, pues solo hay una pieza que presenta un cierto formato humorístico ("Sílvia on the jungle»). 
La orientación del discurso (positiva, negativa, neutra o indeterminada) de los vídeos es mayoritariamente positiva (la trama e intención autoral trata de resolver un problema de violencia de género). Sólo se han identificado dos ejemplos de carácter negativo o neutro: "Acoso» (universitarios) y «La hipocresía del influencen» (secundaria). En ninguno de los dos casos se aportan alternativas al problema, pero además en "Acoso" el discurso parece enfatizar la dificultad extrema de la huida efectiva o de la denuncia. La situación de acoso tampoco se resuelve más que por el final semi-abierto (perseguidor y perseguida se reencuentran). Esta orientación coincide con los dos únicos casos de estructura declarativa y aséptica de la muestra. El resto de vídeos, en cambio, presentan una estructura reflexiva/interrogativa (vídeos que plantean preguntas o que mueven la conciencia) o imperativa (vídeos que incluyen órdenes que mueven a la acción). Esta última se relaciona con una interpelación directa al espectador y con una orientación del discurso más reactiva. Por la propia naturaleza del encargo (hacer un vídeo relacionado con YouTube), dicha interpelación se incorpora también al final de algunos vídeos de naturaleza reflexiva, a través de la figura del supuesto YouTuber. Es por ello que en seis de los vídeos se reconoce una estructura mixta, mientras que son tres los que presentan una estructura únicamente imperativa y cuatro únicamente reflexiva/interrogativa (los otros dos corresponden a la mencionada estructura declarativa/aséptica).

En relación al tipo de violencia, de acuerdo con los resultados de los grupos de discusión en cuanto a la conceptualización de la violencia de género, los estudiantes de secundaria han abordado mayoritariamente formas de violencia simbólica como los estereotipos de género (y sus consecuencias para ambos sexos), los micromachismos, la cosificación, y la invisibilización histórica de la mujer, y sólo ocasionalmente han tratado el acoso callejero y la violencia física y/o sexual. Entre la primera juventud, los jóvenes universitarios abordan sobre todo el acoso sexual, seguido por formas de violencia física graves (agresión; abuso; una referencia al feminicidio) y simbólicas (violencia verbal; celos; prejuicios y estereotipos de género - también contra los hombres-, y un caso de defensa de la normalización de la homosexualidad femenina). Resulta destacable, además, que varios vídeos en ambas franjas de edad vinculan la violencia de género a otras formas de violencia, como pueden ser la homofobia (en el caso de «Sexismo en el aula» y en «Seamos el cambio») o el racismo y el clasismo (en el caso de "Mente abierta» y, de nuevo, "Seamos el cambio»).

En las producciones de ambas franjas de edad los personajes son escasos (y representan a alumnos, profesorado, compañeros, parejas sentimentales, padres), o incluso inexistentes (voz en off). Algún personaje puede asumir un rol de narrador, sobre todo cuando se opta por una introducción o epílogo del vídeo (narradorpersonaje). En el extremo opuesto, el narrador omnisciente acompaña algunas recreaciones ficcionales mientras que los collage optan por un narrador interno fuera de campo. Cabe recordar que, tal como señalan Scolari y Fraticelli (2016), la edición deviene en muchos casos una suerte de enunciador extradiegético que, particu- 
larmente en el caso del género del vlog, acompaña, corrige o incluso contradice al YouTuber (esto es especialmente explícito en el vídeo "Si eres una YouTuber...»).

Por último, respecto al tratamiento del espacio-tiempo, la mayoría de vídeos simulan una situación de cierta cotidianeidad y proximidad (escuela, calle, domicilio...). De acuerdo con sus posibilidades (escaso tiempo de grabación e imposibilidad de abandonar el centro escolar), los vídeos de los institutos muestran una escenografía más sencilla (muchas veces con fondos neutros) y una uniformidad en los espacios (mayoritariamente interiores y en un aula), en relación a los vídeos realizados por estudiantes universitarios, en los que un mayor tiempo de rodaje y de montaje permite una mayor recreación en la puesta en escena y algún leve juego de orden temporal.

\section{Discusión y conclusiones}

Los resultados de esta investigación confirman que YouTube puede ser para las nuevas generaciones un instrumento a favor de la sensibilización y prevención de la violencia de género. En relación al sentido que adolescentes y jóvenes otorgan a YouTube, hemos podido comprobar que es una plataforma perfectamente integrada en su vida cotidiana. Sin embargo, hay diferencias en las percepciones de uso según la edad/nivel de estudios y según el género. No solo el contenido y los YouTubers preferidos son diferentes según este último, sino que persiste el prejuicio según el cual la falsedad es un atributo de las YouTubers femeninas. Además, las participantes mujeres se encuentran más familiarizadas con los y las YouTubers con perspectiva de género que los hombres (una tendencia más marcada entre los adolescentes que entre los jóvenes).

Tanto en la identificación de los YouTubers con sensibilidad de género como en la realización de los vídeos se detecta una asociación de la lucha feminista con la de los derechos LGTBQ. Ello podría indicar que los alumnos han integrado (seguramente de forma inconsciente) el concepto de interseccionalidad (Crenshaw, 1980), además de confirmar el impacto entre la audiencia de los contenidos sobre sexualidad y/o identidad de género en YouTube, observada en investigaciones previas (Pérez-Torres et al., 2018).

A pesar de la sensibilidad social sobre el machismo, esta no es la primera forma de violencia que los estudiantes reconocen cuando son interpelados sobre la presencia de la misma en redes sociales. De manera espontánea, en los grupos de discusión emergen primero ejemplos de maltrato animal, de discriminación racial o de violencia interpersonal directa. De hecho, se ha observado que entre los más jóvenes se llega a un consenso sobre qué es la violencia de género después de recurrir a diversos ejemplos. Esta estrategia, que en gran medida puede atribuirse a la progresiva capacidad de conceptualización que acompaña a la madurez intelectual, tiene un cierto paralelismo en el tipo de temas y en el abordaje que se hace de la violencia de género 
en los vídeos. Si bien entre los universitarios se observa una mayor variedad de tipos de violencia de género, no deja de ser relevante que sea entre los estudiantes de secundaria donde hay una mayor representación de formas sutiles de violencia, situadas en ese $90 \%$ invisible del iceberg de la violencia de género. A pesar de que en los focus group se ha identificado la concepción ambivalente de dos estudiantes respecto a la violencia de género, según la cual ésta opera en ambas direcciones, ningún vídeo presenta a los varones como víctimas, excepto en el caso de alguno de los vídeos que critican los estereotipos de género. Por otro lado, las formas de la violencia estructural prácticamente no aparecen (desigualdad salarial, techo de cristal...), exceptuando la pieza sobre la falta de (re)conocimiento de las mujeres a la largo de la Historia.

Tanto los vídeos, de orientación mayormente positiva, como las intervenciones en los grupos de discusión muestran que hay un posicionamiento contrario a la violencia de género. Además, ambos reflejan diversidad en la conceptualización de la misma (acoso, estereotipos de género, ausencia de la mujer en la historia, micromachismos...), y proponen una gran variedad de formatos para abordarla desde el audiovisual (ficción, entrevistas, vlogs...).

La orientación metodológica que se ha propuesto en este proyecto, que incluye la decisión de incorporar el Aprendizaje Servicio para favorecer la prosumición de los participantes, ha perseguido precisamente una modalidad de encuentro entre la academia y la sociedad. Sin renunciar a la rigurosidad y al aprendizaje por competencias, se ha integrado el Aprendizaje Servicio como un elemento natural para aproximarnos a los y las adolescentes y abordar conjuntamente la relación que establecen con medios sociales como YouTube. Además, el propósito de otorgar protagonismo a las reflexiones de los y las adolescentes, ha permitido el aprendizaje y el trabajo tanto desde los institutos como desde la universidad sobre una necesidad real como es la prevención de la violencia de género y que se ha articulado en un proyecto conjunto entre investigadoras de comunicación audiovisual y formadoras mediáticas en entornos socioeducativos. El proyecto responde al requerimiento de ser un servicio a la comunidad y se enmarca en los actuales interrogantes sobre el sentido, los valores y las posibilidades de los nuevos medios, en este caso acompañando a la incipiente juventud a generar producciones audiovisuales que colaboren en la erradicación de la violencia de género. Un objetivo posible cuando se confirma que la educación participa en transformar la sociedad en modelos de convivencia más inclusivos y equitativos.

Ayuda y agradecimientos: El proyecto «Propuesta de formación en social media literacy: Talleres para y con adolescentes y jóvenes sobre YouTube con una perspectiva de genero inclusiva» ha recibido la financiación del Pacto de estado contra la violencia de género (2018) del Ministerio de la Presidencia, Relaciones con las Cortes e igualdad.

Agradecemos la participación de los centros educativos y profesorado y especialmente la de los y las jóvenes estudiantes, así como la colaboración de Drac Màgic. 


\section{Referencias bibliográficas}

Ajuntament de Barcelona (2017). Distribució territorial de la renda familiar disponible per càpita a Barcelona 2017. https://bit.ly/2Q7W5Cw

Amnistía internacional (2009). Noticias que salvan vidas: manual periodístico para el abordaje de la violencia contra las mujeres. Buenos Aires: A.I. Argentina-Asoc. Civil Pro Amnistía. https://bit.ly/39rNdiI

Amnistía internacional (2019). Violencia de género en España. https://bit.ly/352SJVK. Consulta el 17 de diciembre de 2019.

Annette, J. (2005). «Character, civic renewal and service learning for democratic citizenship in higher education». British Journal of Educational Studies, 53, 3, 326-340.

Aran, S; De Puig, I. (2011). Violencia: tolerancia cero. Programa educativo: Secundaria. Barcelona: Obra social «la Caixa». https://tinyurl.com/rkd6uak

Aran-Ramspott, S., Fedele, M., \& Tarragó, A. (2018). YouTubers' social functions and their influence on pre-adolescence. [Funciones sociales de los Youtubers y su influencia en la preadolescencia]. Comunicar, 57, 71-80. https://doi.org/10.3916/C572018-07

Arandia, M., Alonso, M.J. y Martínez, I. (2010). «La metodología dialógica en las aulas universitarias». Revista de educación, 352, 309-329.

Araüna, N., Tortajada, I. y Willem, C. (2019). «Discursos feministes i vídeos de youtuberes: límits i horitzons de la politització jo-cèntrica». Quaderns del CAC 45, vol. XXII, 25-34.

Boyd, D. (2014). It's complicated: The social lives of networked teens. New Haven (Connecticut): Yale University Press. https://bit.ly/2IxtlQI

Buckingham, D. (2003). Media education. Literacy, learning and contemporary culture. London: Polity Books.

Buckingham, D. (2008). Youth, identity and digital media. Cambridge, MA: The MIT Press.

Caballero-Gálvez, A., Tortajada, I. y Willem, C. (2017). «Autenticidad, marca personal y agencia sexual: el posfeminismo lésbico en YouTube». Investig. Fem (Rev.) 8 (2): 353368.

Crenchaw, K.W. (1989). «Demarginalizing the intersection of race and sex: a black feminist critique of anti-discrimination doctrine, feminist theory and anti-racist politics». University of Chicago Legal Forum, 1989(1), 139-16. https://bit.ly/39tOQwC

Eyler, J. y Giles, D.E. Jr. (1999). Where's the Learning in Service-Learning? San Francisco: Jossey-Bass Higher and Adult Education Series.

Fernández Alonso, M. (2003) Violencia doméstica. Madrid: Ministerio de Sanidad y consumo. https://bit.ly/39q0Y1v

Fernández-de-Arroyabe-Olaortua, A.; Lazkano-Arrillaga, I. y Eguskiza-Sesumaga, L. (2018). «Digital natives: Online audiovisual content consumption, creation and dissemination». [Nativos digitales: Consumo, creación y difusión de contenidos audiovisuales online]. Comunicar, 57, 61-69. https://doi.org/10.3916/C57-2018-06 
Ferrés, J. y Piscitelli, A. (2012). «Media competence. Articulated proposal of dimensions and indicators». [La competencia mediática: Propuesta articulada de dimensiones e indicadores]. Comunicar, 38, 75-82. https://doi.org/10.3916/C38-2012-02-08

Gewerc, A., Fraga, F. y Rodes, V. (2017). «Niños y adolescentes frente a la competencia digital. Entre el teléfono móvil, youtubers y videojuegos». Revista Interuniversitaria de Formación del Profesorado, 89, 171-186. https://bit.ly/2rBmpZf

Halliday, M. (2004). An Introduction to Functional Grammar. London: Edward Arnold.

Linares-Bahillo, E.; Aristegui-Fradua, I. y Beloki-Marañón, U. (2019). «YouTube, una plataforma para la (in)formación, relación, comunicación, diversión, y gestión de identidades (de género) en la natividad digital». Revista Mediterránea de Comunicación, 10(1), 55-70. https://doi.org/10.14198/MEDCOM2019.10.1.18

Livingstone, S. (2008). «Taking risky opportunities in youthful content creation: Teenagers' use of social networking sites for intimacy, privacy and self-expression». New Media $\mathcal{E}$ Society, 10(3), 393-411. https://doi.org/10.1177/1461444808089415

López-Vidales, N. y Gómez-Rubio, L. (2015). «Análisis y proyección de los contenidos audiovisuales sobre jóvenes y drogas en YouTube». Estudios sobre el Mensaje Periodístico, 21(2), 863-881. https://doi.org/10.5209/rev_ESMP.2015.v21.n2.50889

Montes-Vozmediano, M., García-Jiménez, A. y Menor-Sendra, J. (2018). «Teen videos on YouTube: Features and digital vulnerabilities». [Los vídeos de los adolescentes en YouTube: Características y vulnerabilidades digitales]. Comunicar, 54, 61-69. https:// doi.org/10.3916/C54-2018-06

Obra Social «La Caixa» (2006). Violencia: tolerancia cero. Curso de formación. La prevención de la violencia de género en el aula. https://bit.ly/39j25jo

Pérez-Torres, V., Pastor, Y. y Abarrou, S. (2018). «Los YouTubers y la construcción de la identidad adolescente». Comunicar XXVI (55), 61-70. https://doi.org/10.3916/C552018-06

Pibernat, M. (2017). «¿Nuevas socializaciones, viejas cuestiones? Adolescencia y género en la era audiovisual». Investigaciones Feministas, 8(2), 529-544. https://doi.org/10.5209/ INFE.54976

Pires, F.; Masanet, M. J. y Scolari, C. (2019). «What are teens doing with YouTube? Practices, uses and metaphors of the most popular audio-visual platform». Information, Communication \& Society, DOI: 10.1080/1369118X.2019.1672766

Puig, J.M. (2012). Compromís cívic i aprenentatge a la universitat: Experiències i institucionalització de l'aprenentatge servei. Barcelona: Graó.

Puig, J.M, Martín, X. y Batlle, R. (2008). Cómo iniciar un proyecto de aprendizaje y servicio solidario. Bilbao: Zerbikas.

Scolari, C. y Fraticelli, D. (2016). «Nuevos sujetos mediáticos en el ecosistema de medios». $V$ Congreso AsAECA, Buenos Aires.

Scolari, C. (2016). "Alfabetismo transmedia: estrategias de aprendizaje informal y competencias mediáticas en la nueva ecología de la comunicación». Telos: Revista de pensamiento sobre Comunicación, Tecnología y Sociedad, 193, 13-23. https://bit.ly/358g8oN 
Simonsen, T.M. (2012). Identity formation on YouTube (Doctoral Dissertation). Aalborg: Aalborg University.

Tortajada, I. Caballero-Gálvez, A. y Willem, C. (2019). «Contrapúblicos en YouTube : el caso del colectivo trans». El profesional de la información, v.28, n.6, e280622. https://doi. org/10.3145/epi.2019.nov.22

Viñals, A.; Abad, M. y Aguilar, E. (2014). «Jóvenes conectados: Una aproximación al ocio digital de los jóvenes españoles». Comunication Papers, 3(4), 52-68. https://bit. ly/2KgpcOh

Wotanis, L. y McMillan, L. (2014). «Performing Gender on YouTube. How Jenna Marbles negotiates a hostile online environment». Feminist Media Studies, 14(6), 912-928. https://doi.org/10.1080/14680777.2014.882373 